\title{
Perfil de atendimento em unidades de pronto atendimento em um município do oeste paranaense
}

\section{Profile of care in emergency care units in a municipality in western Paraná}

Thainá Klosowski Kulicz¹, Kurt Juliano Sack Orejuela Uscocovich²

1. ORCID: https://orcid.org/0000-0002-6125-1378. Acadêmica do décimo período de Medicina no Centro Universitário Fundação Assis Gurgacz, Cascavel, Paraná, Brasil. E-mail: thainakulicz2@hotmail.com

2. ORCID: https://orcid.org/0000-0002-6119-6700. Médico da Família e Comunidade. Docente do curso de Medicina no Centro Universitário Fundação Assis Gurgacz, Cascavel, Paraná, Brasil. E-mail: kurtsou@yahoo.com.br

CONTATO:Autor correspondente: Thainá Klosowski Kulicz | Endereço: Rua Riachuelo, 2420, Centro, Cascavel-PR, CEP: 85.812-110 | Telefone: (42) 99972-5722 | E-mail: thainakulicz2@hotmail.com

RESUMO O presente estudo avaliou o perfil de atendimento de pacientes que procuram as Unidades de Pronto Atendimento (UPAs) em um município do oeste do Paraná, segundo o Protocolo de Manchester. Com isso, objetivou-se caracterizar a demanda de usuários que buscam suporte na Atenção Secundária e identificar se o uso desses serviços ocorre conforme é determinado pela Política Nacional de Atenção às Urgências e Emergências (PNAU). Trata-se de uma pesquisa retrospectiva e transversal de abordagem quantitativa. Para determinação de resultados foram selecionadas pessoas, de todas as idades, atendidas em 2 UPAs do município durante o período de janeiro até dezembro de 2019. A amostra válida do estudo foi de 144.459 atendimentos realizados e classificados de acordo com a classificação de risco. Constatou-se que a maioria dos atendimentos prestados foram classificados como verde e amarelo, diferentemente do que é proposto pela lei.

DESCRITORES: Serviços Médicos de Emergência. Triagem. Níveis de Atenção à Saúde. Atenção Secundária à Saúde. 


\begin{abstract}
This study evaluated the care profile of patients who seek Emergency Care Units (UPAs) in a city in western Paraná, according to the Manchester Protocol. Thus, the objective was to characterize the demand of users who seek support in Secondary Care and to identify whether the use of these services occurs as determined by the National Policy for Urgent and Emergency Care (PNAU). This is a retrospective and transversal study with a quantitative approach. To determine the results, people of all ages were selected, attended in 2 UPAs in the municipality during the period from January to December 2019. The valid sample of the study was 144,459 consultations performed and classified according to the risk classification. It was found that most of the services provided were classified as green and yellow, unlike what is proposed by law.
\end{abstract}

DESCRIPTORS: Emergency Medical Services. Triage. Health Care Levels. Secondary Care. 


\section{INTRODUÇÃO}

Sistema Único de Saúde (SUS) é composto por uma série de serviços articulados que se complementam e tem como objetivo atender de maneira eficiente toda a população que necessita de suporte ${ }^{1}$. Os serviços oferecidos pelo SUS são separados em níveis de atenção, sendo elas primária, secundária e terciária. Essa estrutura visa organizar as ações e serviços oferecidos pelo sistema ${ }^{1,2}$.

A Atenção Primária à Saúde, preferencial porta de entrada ao SUS, é composta pelas Unidades Básicas de Saúde (UBS) e Unidades de Saúde da Família (USF). Sua função é realizar atendimentos de baixo risco e também atuar na promoção, prevenção, diagnóstico e tratamento precoce dos pacientes. É essencial fazer um acompanhamento contínuo desses pacientes afim de evitar futuras complicações preveníveis ${ }^{3}$. Esse nível de complexidade é capaz de atender e resolver mais de $80 \%$ dos problemas de saúde da população 4 .

Os serviços de média complexidade são realizados por estabelecimentos intermediários entre as UBS e a Rede Hospitalar, as Unidades de Pronto Atendimento (UPAs). São, basicamente, hospitais simplificados que funcionam 24 horas por dia em todos os dias da semana. $\mathrm{O}$ acolhimento dos pacientes é realizado com base na classificação de risco o que permite rápido suporte àqueles que necessitam de conduta imediata. Portanto, o que dita a priorização do atendimento é a gravidade do caso e não a ordem de chegada a Unidade ${ }^{5,6}$. Atualmente, o Sistema de Triagem de Manchester (STM) é o protocolo de classificação por cor mais utilizado nas UPAs do Brasil. Essas estruturas são regidas pela Política Nacional de Urgências e Emergências (PNAU), publicada pelo Ministério da Saúde em $2003^{6}$.

A alta complexidade é designada à Atenção Hospitalar que proporciona uma série de terapias e procedimentos altamente especializados, além de envolverem alta tecnologia ${ }^{6}$.

A participação da população é um dos principais pilares para o funcionamento adequado do SUS ${ }^{1}$. É importante que os usuários entendam o sistema, saibam a função de cada complexidade de saúde, conheçam o acolhimento com classificação de risco e façam o uso correto dos serviços oferecidos para que não ocorra a desarticulação dos diferentes níveis de atenção ${ }^{7}$.

Na prática, a grande maioria dos utilizadores do sistema desconhecem a organização e o funcionamento do SUS ${ }^{8,9}$. Devido a essa falta de informação e concepção ${ }^{7-9}$, é observado que as pessoas não seguem a hierarquização dos serviços de saúde de acordo com a complexidade do atendimento, como é estabelecido pela lei².

Mesmo com a ampliação da oferta de serviços de Atenção Básica desde 1990, é comum ver grande parte da população em busca de consultas não urgentes na Atenção Secundária $^{10}$. Isso dificulta o atendimento integral ao paciente, tornando a assistência fragmentada. 
As UPAs estão sendo a escolha, como porta de entrada ao sistema, para solucionarem problemas de saúde sensíveis de serem resolvidos pela Atenção Primária ${ }^{11-15}$. Os pacientes procuram essas Unidades pela rápida e fácil resolução dos seus problemas, além delas oferecem mais recursos e opções de exames complementares ${ }^{16}$. Como consequência desse uso equivocado da Atenção Secundária como o primeiro acesso aos serviços de saúde é notado a superlotação nessa complexidade, falta de leitos, aumento das filas de espera e estresse tanto profissional quanto do usuário ${ }^{17}$.

Neste sentido, devido a importância do reconhecimento do uso inadequado desses serviços, o estudo analisou o perfil de atendimento, de acordo com o Protocolo de Manchester, realizado nas UPAs de um município do oeste paranaense. O presente artigo se propõe a caracterizar a demanda de pacientes que buscam suporte na Atenção Secundária e identificar se o uso desses serviços ocorre conforme determinado pela Política Nacional de Atenção às Urgências e Emergências (PNAU).

\section{MÉTOdO}

Este estudo foi baseado metodologicamente como uma pesquisa retrospectiva e transversal de abordagem quantitativa.

A população de estudo foi composta por pessoas, de todas as idades, que buscaram atendimento em 2 UPAs de um município do oeste do Paraná e foram classificadas em sua admissão conforme o Protocolo de Manchester, no período de 1 de janeiro de 2019 até 31 de dezembro 2019. Os dados foram coletados através da análise de prontuários eletrônicos fornecidos pelo sistema de informação utilizado pela Secretária Municipal de Saúde do munícipio.

O Sistema de triagem de Manchester (STM), criado em 1994 na Inglaterra e implantado pela primeira vez no Brasil em 2008, classifica a gravidade do caso do paciente e determina o tempo máximo que o usuário pode aguardar pelo atendimento ${ }^{18,19}$. Sua classificação permite a avaliação dos pacientes por meio dos critérios clínicos e não pela ordem de chegada ao serviço. O protocolo é composto por 5 níveis de prioridade: nível 1 (vermelho) são pacientes emergentes de cuidado e devem receber atendimento médico imediato; nível 2 (laranja) são pacientes muito urgentes e precisam de atendimento em até 10 minutos; nível 3 (amarelo) são pacientes urgentes e necessitam de avaliação médica em até 60 minutos; nível 4 (verde) são pacientes pouco urgentes que devem ser atendidos em até 120 minutos e, por fim, nível 5 (azul) são os pacientes não urgentes e que podem esperar o atendimento até 240 minutos $^{19}$.

O Projeto de Pesquisa foi submetido ao Comitê de Ética em Pesquisa com Seres Humanos do Centro Universitário FAG e aprovado dia 29 de fevereiro de 2020 pelo CAAE número 26536719.1.0000.5219. Por se tratar de uma pesquisa com seres hu- 
manos, este estudo está em cumprimento com a Resolução 466/12 do Conselho Nacional de Saúde.

\section{RESULTADOS}

Em uma observação geral, foram registrados 144.459 atendimentos totais nas UPAs Brasília e Veneza do município de Cascavel-PR durante o período de 1 de janeiro de 2019 até 31 de dezembro de 2019.

Quando dividimos conforme a classificação de risco, a maior parte dos pacientes foi classificado na categoria verde ou pouco urgente, correspondendo a 101.302 atendimentos $(70,12 \%)$, seguido da cor amarelo ou urgente com 36.047 (24,95\%), azul com 4.252 (2,94\%), laranja com $2.756(1,90 \%)$ e, por fim, a cor vermelha ou emergente com 102 atendimentos $(0,07 \%)$.

Em uma análise isolada, foram 68.066 atendimentos na UPA Brasília e 76.393 atendimentos na UPA Veneza. Quanto a classificação de risco, foram verificados na UPA Brasília 47.945 atendimentos verdes, 16.073 amarelos, 2.788 azuis, 1.223 laranjas e 37 vermelhos. Já na UPA Veneza foram contabilizadas 53.357 consultas verdes, 19.974 amarelos, 1.464 azuis, 1.533 laranjas e 65 vermelhos. Os resultados encontram-se no Gráfico 1.

Gráfico 1: Atendimentos conforme classificação de risco realizados no período de janeiro até dezembro de 2019 nas UPAs Brasília e Veneza de Cascavel-PR.

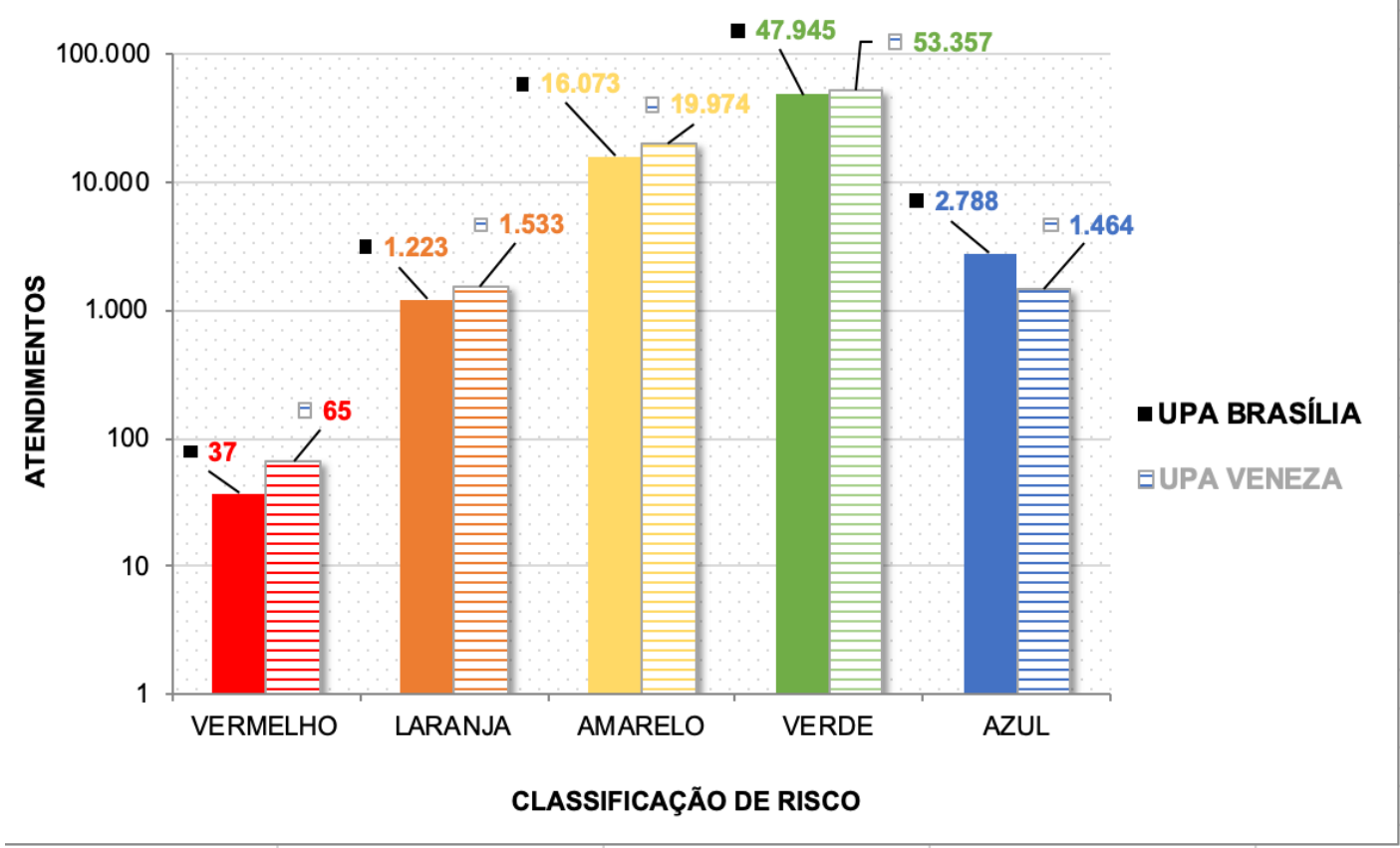

Fonte: Elaborado pelos autores (2020). 


\section{DISCUSSÃO}

Existe uma divergência entre a proposta original de que atendimentos de média complexidade devem ser prestados pelas UPAs, como estabelecido pela PNAU, e o atual cenário de atendimentos realizado por essas Unidades.

As UPAs têm como propósito acolher pacientes em situação de urgência e emergência, prestar atendimento resolutivo e qualificado aos quadros agudos ou crônicos agudizados de natureza clínica e prestar o primeiro suporte aos casos de natureza cirúrgica e de trauma ${ }^{6}$. Apesar da especificidade destes serviços para acolher os casos de saúde de média gravidade, a realidade é marcada pela inversão de fluxo de usuários entre a Atenção Básica e a Atenção Secundária.

As UPAs do munícipio estudado seguem o acolhimento conforme protocolo de Manchester, no qual os casos classificados como emergência (vermelho) necessitam de atendimento imediato; muito urgente (laranja) devem ser atendidos em até 10 minutos; urgente (amarelo) podem ser atendidos em até 60 minutos; pouco urgentes podem aguardar atendimento por até 120 minutos e não urgentes que podem aguardar atendimento por até 240 minutos $^{18}$. Observou-se que apenas $0,07 \%$ dos usuários foram classificados como emergência (vermelho) e 24,95\% como urgência (amarelo), mostrando uma distorção do verdadeiro papel das UPAs, o qual seria de atender urgências e emergências.

Entre os pacientes pesquisados evidenciou-se que houve mais atendimentos às pessoas classificadas como verde $(70,12 \%)$, este dado se assemelha ao de outros estudos realizados por pesquisadores brasileiros ${ }^{14,15}$. Em estudos semelhantes, obtiveram como resultado que $67 \%$ dos atendimentos em uma Unidade de emergência referenciada de um hospital de ensino ${ }^{14}$ e $51 \%$ dos atendimentos prestados na UPA de outro munícipio avaliado $^{15}$ foram classificados como verde. Os autores associam esta alta demanda de atendimentos pouco urgentes a uma falta de orientação da população sobre a utilização correta dos serviços disponíveis e a uma carência da concepção concreta do que seriam casos de urgência e emergência.

Os Órgãos Públicos não conseguem informar sobre os serviços ofertados e o caminho que o usuário deve percorrer dentro do sistema ${ }^{8}$. A falta de conhecimento da população ${ }^{7-9,14,15}$ é um sério fator que gera a desarticulação dos níveis de atenção à saúde. Por não saberem como o SUS funciona, os usuários dirigem-se ao serviço de saúde que mais os convêm.

Uma revisão sistemática realizada para compreender as razões que levam o paciente a buscar o serviço de Pronto Atendimento (PA), quando estes poderiam ser atendidos na Atenção Básica, revelou que a restrição de horário de funcionamento das UBS, a dificuldade de encontrar agenda disponível na UBS, considerar que poderia necessitar de um 
atendimento de urgência e a maior disponibilidade de recursos no PA do que na UBS são fatores que influenciam a decisão de procurar por este tipo de serviço ${ }^{12}$. Dessa forma, é evidente que os usuários preferem a rápida resolução dos seus problemas e por isso dirigem-se aos $\mathrm{PA}^{12-16}$.

O uso errôneo dos serviços da Atenção Secundária é preocupante, uma vez que essa busca crescente por consultas causa uma demanda maior do que a capacidade de atendimentos e, consequentemente, retrata um cenário de superlotação e falta de leitos nas UPAs $^{17-20}$. Além disso, há a desorganização das Unidades, elevação das despesas ao sistema e geração de gastos desnecessários, o que resulta em uso pouco racional dos recursos disponíveis ${ }^{20}$.

Outro problema que ocorre é um atendimento médico que não consegue estabelecer vínculo dos usuários com os serviços, fator fundamental para garantir cuidados contínuos em saúde. Naquele momento, os pacientes só recebem tratamento para aliviar sintomas pontuais e esquecem da importância de um acompanhamento médico para prevenir novas complicações e outras doenças ${ }^{21}$.

A nossa sociedade requer mudanças culturais. As pessoas precisam entender a estrutura hierárquica dos serviços médicos, a importância da articulação dos níveis de saúde e a importância do acompanhamento contínuo ${ }^{14}$.

A partir dos achados do presente estudo, é importante traçar metas para melhorar os serviços. Sugere-se a implementação de campanhas e políticas educacionais direcionadas à população com o propósito de orientá-los sobre o SUS, a articulação dos diferentes níveis de complexidade do sistema, o conceito da classificação de risco e como usar de maneira correta os serviços oferecidos.

\section{CONSIDERAÇÕES FINAIS}

O presente estudo permitiu evidenciar que grande parte dos atendimentos prestados pelas UPAs avaliadas não necessitavam dos serviços de Atenção Secundária e, em sua maioria, poderiam ser resolvidos pela Atenção Básica. Constatou-se que a maioria dos atendidos foram classificados, de acordo com o Protocolo de Manchester, como verde e amarelo que são considerados, respectivamente, pacientes pouco urgentes e urgentes. Contudo, vale lembrar que as UPAs foram criadas com objetivo de atender situações de urgência e emergência.

Portanto, os resultados apresentados corroboram a hipótese desse estudo de que o serviço prestado pelas UPAs não está diretamente relacionado à sua proposta original $\mathrm{e}$ também sustenta as pesquisas realizadas por outros pesquisadores que mostraram que grande parte dos atendimentos realizados pelas UPAs são de baixa complexidade. 
Na perspectiva da consolidação dos princípios do SUS, é importante compreender essa realidade em nosso meio no sentido de avaliar as atuais necessidades de reorganização do sistema de saúde. Definir a demanda e o perfil da população que procura a Atenção Secundária, proporciona o reconhecimento dos usos inadequados do serviço e a necessidade de reprimi-los, a fim de evitar superlotação das Unidades e estresse profissional e do paciente.

Diante do exposto, há necessidade de esclarecer aos usuários sobre o SUS, a função de cada complexidade de saúde, o conceito da classificação de risco e, mais importante, como fazer o uso correto dos serviços oferecidos. Sugere-se a elaboração de campanhas e políticas educacionais direcionadas à população cascavelense com o intuito de informá-los e instruí-los.

\section{REFERÊNCIAS}

1. Brasil. Lei $n^{\circ} 8.080$, de 19 de setembro de 1990. Dispõe sobre as condições para a promoção, proteção e recuperação da saúde, a organização e o funcionamento dos serviços correspondentes e dá outras providências. Brasília: Diário Oficial da União; 1990. Disponível em: http://www.planalto.gov.br/ccivil_03/ leis//8080.htm

2. Brasil. Decreto $n^{\circ} 7.508$, de 28 de junho de 2011. Regulamenta a Lei $n^{\circ} 8.080$, de 19 de setembro de 1990, para dispor sobre a organização do Sistema Único de Saúde - SUS, o planejamento da saúde, a assistência à saúde e a articulação interfederativa, e dá outras providências. Brasília: Diário Oficial da União; 2011. Disponível em: http://www.planalto.gov.br/ccivil_03/_ato2011-2014/2011/decreto/d7508.htm

3. Brasil. Portaria $n^{\circ} 2.436$, de 21 de setembro de 2017. Aprova a Política Nacional de Atenção Básica, estabelecendo a revisão de diretrizes para a organização da Atenção Básica, no âmbito do Sistema Único de Saúde (SUS). Brasília: Ministério da Saúde; 2017. Disponível me: https://bvsms.saude.gov.br/bvs/saudelegis/gm/2017/prt2436_22_09_2017.html

4. Paim, J. S. O que é o SUS [Internet]. Rio de Janeiro: Fiocruz; 2015 [citado em 15 jul. 2020]. Disponível em: http://www.livrosinterativoseditora.fiocruz.br/sus/

5. Brasil. Portaria $n^{\circ} 10$, de 3 de janeiro de 2017. Redefine as diretrizes de modelo assistencial e financiamento de UPA 24h de Pronto Atendimento como Componente da Rede de Atenção às Urgências, no âmbito do Sistema Único de Saúde. Brasília: Ministério da Saúde; 2017. Disponível em: http://bvsms.saude. gov.br/bvs/saudelegis/gm/2017/prt0010_03_01_2017.html

6. Brasil. Portaria $n^{0} 1.600$, de 7 de julho de 2011. Reformula a Política Nacional de Atenção às Urgências no Sistema Único de Saúde (SUS). Brasília: Ministério da Saúde; 2011. Disponível em: http://bvsms.saude.gov.br/bvs/saudelegis/gm/2011/prt1600_07_07_2011.html

7. Tostes PCM, Celestino CSM, Cruz KM, Chevitarese L. Acolhimento e classificação de risco em Unidade de Pronto Atendimento (UPA): uma abordagem educativa. Rev. Rede cuid. saúde [Internet]. 2017 [citado em 21 jul. 2020];10(1). Disponível em: http://publicacoes.unigranrio.edu.br/index.php/rcs/article/ view/3289

8. Hamada RKF, Almeida VOC, Brasil ICF, Souza SG, Luzia RAB, Campos EMS, Leite ICG. Conhecendo o Sistema Único de Saúde: um olhar da população. Rev. APS. 2018 Dez;21(4):504-15. doi: https://doi. org/10.34019/1809-8363.2018.v21.16459

9. Kawashima MCS, Ferreira MV, Silva PC, Boaventura AP, Almeida JB. O Desconhecido SUS: O conhecimento da população de uma cidade do Vale do Paraíba. $11^{\circ}$ Encontro Latino Americano de Iniciação Científica; 2007 Out 18-19; São José dos Campos - SP. São José dos Campos: Universidade do Vale do Paraíba; 2007. Disponível em: http://www.inicepg.univap.br/cd/INIC_2007/trabalhos/saude/inic/INICG00420_010.pdf 
10. Machado CV, Salvador FGF, O'dwyer G. Serviço de Atendimento Móvel de Urgência: análise da política brasileira. Rev. saúde pública [Internet]. 2011 [citado em 8 ago. 2020]; 45(3): 519-528. Disponível em: https://www.scielosp.org/article/rsp/2011.v45n3/519-528/

11. Gomide MFS, Pinto IC, Figueiredo LA. Acessibilidade e demanda em uma Unidade de Pronto Atendimento: perspectiva do usuário. Acta Paul Enferm. [Internet] 2012 [citado em 18 ago. 2020];25(spe2):19-25. doi: http://dx.doi.org/10.1590/S0103-21002012000900004

12. Caccia-Brava MCG, Pereira MJB, Rocha JSY, Martinez EZ. Pronto-atendimento ou atenção básica: escolhas dos pacientes no SUS. Medicina (Ribeirão Preto) [Internet]. 2011 [citado em 18 ago. 2020];44(4):34754. Disponível em: http://www.revistas.usp.br/rmrp/article/view/47446

13. Cassettari SSR, Mello ANSF. Demand and type of care provided in emergency services in the city of Florianópolis, Brazil. Texto Contexto Enferm. [Internet]. 2017 [citado em 18 ago. 2020];26(1). doi: https:// doi.org/10.1590/0104-07072017003400015

14. Oliveira, MD. Perfil da demanda de atendimentos realizados pela Unidade de Pronto Atendimento (UPA) de Tramandaí/RS [trabalho de conclusão de curso]. Porto Alegre: Universidade Federal do Rio Grande do Sul: Escola de Administração, Curso de Especialização em Gestão em Saúde; 2019. Disponível em: http://hdl.handle.net/10183/201878

15. Oliveira GN, Silva MFN, Araujo IEM, Carvalho-Filho MA. Perfil da população atendida em uma unidade de emergência referenciada. Rev. latinoam. enferm. [Internet]. Maio-jun 2011 [citado em 19 ago. 2020];19(3):[09 telas]. Disponível em: https://www.scielo.br/pdf/rlae/v19n3/pt_14

16. Marques GQ, Lima MADS. Demandas de usuários a um serviço de pronto atendimento e seu acoIhimento ao sistema de saúde. Rev. latinoam. enferm. [Internet]. Jan-fev 2007 [citado em 25 ago. 2020]; 15(1): 13-19. Disponível em: https://www.scielo.br/pdf/rlae/v15n1/pt_v15n1a03.pdf

17. Siqueira ILCP. Qualidade em serviço de emergência. In: Sallum AMC, Paranhos WY. O enfermeiro e as situações de emergência. 2. ed. São Paulo: Atheneu; 2010. p. 25-34.

18. Coutinho AAP, Cecílio LCO, Mota JAC. Classificação de risco em serviços de emergência: uma discussão da literatura sobre o Sistema de Triagem de Manchester. Rev. Med. Minas Gerais [Internet]. Abr-Jun 2012 [citado em 12 set. 2020]; 22(2): 188-198. Disponível em: http://rmmg.org/artigo/detalhes/101\#

19. Mackway-Jones K, Marsden J, Windle J. Emergency triage: manchester triage group [Internet]. 3a ed. Oxford: John Wiley \& Sons, Ltd.; 2014 [citado em 12 set. 2020]. Disponível em: https://edisciplinas.usp.br/ pluginfile.php/3639780/mod_folder/content/0/Emergency_Triage.pdf?forcedownload=1

20. Azevedo ALCS, Pereira AP, Lemos C, Coelho MF, Chaves LDP. Organização de serviços de emergência hospitalar: uma revisão integrativa de pesquisas. Rev. eletrônica enferm. [Internet]. 2010 [citado em 18 set. 2020]; 12(4): 736-45. Disponível em: https://revistas.ufg.br/fen/article/view/6585/8497

21. Stein AT, Harzheim E, Costa M, Busnello E, Rodrigues LC. The relevance of continuity care: a solution for the chaos in the emergency services. Fam Pract. 2002 Apr; 19(2): 207-10. Acesso em 9 out 2020. doi: https://doi.org/10.1093/fampra/19.2.207 